\title{
CAROTENOIDS IN MAN. III. THE MICROSCOPIC PATTERN OF FLUORESCENCE IN ATHEROMAS, AND ITS RELATION TO THEIR GROWTH ${ }^{1}$
}

\author{
BY DAVID H. BLANKENHORN ${ }^{2}$ AND HERBERT BRAUNSTEIN
}

\author{
(From the Metabolism Laboratory, Department of Medicine, and the Department of Pathology, \\ College of Medicine, University of Cincinnati, The Cincinnati General Hospital,
} Cincinnati, Ohio)

(Submitted for publication May 27, 1957; accepted October 3, 1957)

In a previous study of the carotenoid content of human aortic atherosclerosis it was concluded by Blankenhorn, Freiman, and Knowles that these dietary lipids accumulate in direct proportion to the extent and relative age of the lesions in which they occur. A series of graded aortic segments was assayed, and it was found that increased severity of atherosclerosis was accompanied by a parallel increase in the concentration of carotenoids (1). Exact location of these pigments in relation to the microscopic anatomy of atherosclerotic lesions should aid in evaluation of the role of exogenous lipids of this class in the initiation of atheromas and their continued growth. It seemed possible that this might be accomplished by fluorescence microscopy, since carotenoids and vitamin A, on exposure to ultraviolet light, produce a characteristic pale green fluorescence by which they can be exactly localized and roughly quantitated in tissue sections (2). Although Popper (2), and Hamperl (3), who performed surveys of the fluorescence in human tissue, reported no evidence of carotenoids or vitamin $\mathrm{A}$ in atheromas, it was decided to reinvestigate this matter in view of the findings of Blankenhorn, Freiman, and Knowles. The surveys of Popper and Hamperl were performed on tissues fixed in formalin, a procedure known to diminish the fluorescence of carotenoids and vitamin A. Therefore, in the study here reported unfixed atheromas were examined. Green fluorescence was found and evidence will be presented to show that this fluorescence is a valid means of localizing carotenoids and/or

\footnotetext{
1 Supported in part by grants-in-aid from the American Heart Association, the Youngstown Area Heart Association, Youngstown, Ohio, and the John R. Stark Research Fund.

2 Present address : Department of Medicine, College of Medicine, University of Southern California, Los Angeles, Calif.
}

vitamin $\mathrm{A}$ in atheromatous lesions. As a class, early lesions show little pale green fluorescence, whereas more advanced lesions consistently have this fluorescence distributed through their lipidbearing areas in an apparently random manner. These findings are in accord with the previously published data from chemical assay (1) and provide further evidence that those lipids which accumulate as atherosclerotic lesions increase in size and relative age include certain dietary lipids.

\section{MATERIALS AND METHODS}

Twenty whole aortas obtained at autopsy were examined after storage for short periods of time at refrigerator temperature. The 42 plaques studied were of Grades II to IV, according to a published grading scheme which assigns a grade of IV to the most advanced lesions of atherosclerosis (1). Unfixed frozen sections 15 microns thick, mounted in water on glass slides, were examined with ultraviolet light, and adjacent serial sections, stained with Oil Red $O$ and hematoxylin and eosin, were studied with visible light. A representative segment of each plaque was also fixed in formalin, embedded in paraffin for sectioning, and stained with hematoxylin and eosin. The most satisfactory procedure evolved to date for localizing the fluorescence found in plaques has been by comparison with Oil Red $O$ stained frozen sections at the time of fluorescence microscopy. When necessary, heavily calcified plaques were immersed for periods up to seven days in 10 per cent ethylenediaminetetracetic acid tetrasodium salt (EDTA) at refrigerator temperatures. This has been avoided whenever it appeared possible to cut adequate sections without decalcification, since following this procedure, tissues have a blue cast by ultraviolet light. Efforts to detect green fluorescence are more difficult under these conditions.

The source of ultraviolet light was a Hanovia No. 16106 Mercury Vapor Lamp with a dark red-purple glass filter, which emits maximum energy between $3600 \AA$. and $3700 \AA$. Observations for fluorescence were made with a monocular microscope, screened by a cardboard shield in such a manner as to allow only the substage and mirror to be exposed to ultraviolet light. An alumi- 
TABLE I

The four types of fluorescence found in human atheromas

\begin{tabular}{lcc}
\hline \multicolumn{1}{c}{ Appearance } & Distribution & Source \\
\hline $\begin{array}{c}\text { Pale green, occasionally has a faintly } \\
\text { blue cast. Fades in } 15 \text { to } 30 \text { seconds. }\end{array}$ & $\begin{array}{c}\text { A diffuse, amorphous haze. Occasion- } \\
\text { ally small discrete granules. }\end{array}$ & $\begin{array}{c}\text { Vitamin A } \\
\text { Carotenoids }\end{array}$ \\
$\begin{array}{l}\text { Dim, pale green, or pale pea-green. Oc- } \\
\text { casionally a faintly blue cast. Fades } \\
\text { slowly but not completely, or may not } \\
\text { fade. }\end{array}$ & $\begin{array}{c}\text { A diffuse, amorphous haze. Occasion- } \\
\text { ally small discrete granules. }\end{array}$ \\
$\begin{array}{l}\text { Brilliant yellow or golden brown. Does } \\
\text { not fade. }\end{array}$ & $\begin{array}{c}\text { Discrete, irregular, or oblong crystalline } \\
\text { masses which often appear in clumps. }\end{array}$ \\
$\begin{array}{l}\text { Bright, light blue or bluish-gray. Does } \\
\text { not fade. }\end{array}$ & $\begin{array}{c}\text { Discrete, irregular masses or circlets } \\
\text { which often appear in clumps. }\end{array}$ & $\begin{array}{c}\text { Occasionally a diffuse, amorphous haze. } \\
\text { Sterols? }\end{array}$ \\
\hline
\end{tabular}

nized mirror (American Optical No. 363) was fitted to the microscope. A yellow filter, supplied with this equipment for use in the microscope ocular to prevent corneal injury with prolonged observation, was not used after it was found to obscure areas of faint fluorescence. $\mathrm{Ob}$ servations were carried out in such a way as to limit the exposure of one individual to short periods not more than twice a week.

\section{RESULTS}

\section{A. The fuorescence of atheromas}

With the equipment as described, background illumination is deep purplish-red, and opaque nonfluorescent objects are black. Intact media appears as a brilliant network of sky-blue linear fibers observed against a dark background, suggesting that individual elastic fibers can be visualized against nonfluorescing ground substances. However, blue fluorescence is not specific for elastic tissue since other substances, including lint and cellulose fibers, have this property. Media in areas involved by atheromata does not have this appearance due to loss of elastic fibers. Thus, the extent of medial involvement by atherosclerosis can be readily judged. The effect of pathologic changes other than those of atherosclerosis involving media have not been studied.

In atherosclerotic plaques, fluorescence of four different types is encountered. These types are described in Table I.

Evidence which indicates that the two types of green fluorescence described can be attributed to carotenoids or vitamin $\mathrm{A}$ is provided by the work of Popper and Greenberg (4) and von Querner (5). Additional data supporting this view will be presented below. The source of yellow, golden- brown, and blue fluorescence is not known. The bright yellow and gold bodies have birefringence when examined by polarized light, which suggests that they may be crystallized sterols. When oblong, they are the size and shape of the "cholesterol clefts" seen in atheromas, and they have been observed in areas containing such clefts. Goldenbrown and yellow bodies have been reported by Popper to occur in a number of tissues and have been attributed to lipofuscins (2), but not to sterols. The blue circlets and granular bodies are often found in areas showing focal calcification.

In 42 plaques (17 Grade II, 12 Grade III, 13 Grade IV) the type of fluorescence encountered has been classified and correlated with the pathologic anatomy of the lesions. Each grade of severity presents a characteristic pattern.

Grade II plaques. Plaques of this class are yellow or yellowish-white and opaque upon gross examination. Microscopic examination of stained sections shows that distinct plaque formation is present, with only minimal overlying fibrosis and little or no medial involvement. By ultraviolet light these plaques show very little fluorescence. The media makes up the bulk of the thickness of the aorta, the plaque appearing as a dark area in which faint fluorescence can be observed with some difficulty. The fluorescence present appears as dim, nonfading streaks of green, or homogenous small patches of green, which occasionally appear to fade but are usually so faint that the occurrence of fading cannot be judged accurately. It is found in thin streaks, distributed at random throughout the lipid-bearing areas of the plaque. Occasionally, it may appear concentrated either at the inti- 
mal or medial border of the plaque. Plaques may be encountered which show almost no fluorescence despite the presence of Oil Red $\mathrm{O}$ staining material. It should be noted, however, that such lesions may be yellow by visible light and that carotenoids in trace amounts can be extracted from them, and so it seems possible that with more sensitive equipment fluorescence might be visualized in these plaques.

Scattered, small, round, yellow, or golden-brown bodies are not uncommon and some have a green tint. They may be single or appear in clusters of four or five, and they appear most commonly along the medial (external) border of the plaque. Fibrous areas in the plaque show no fluorescence and appear dark, except for an occasional nonfading yellow granule.

Grade III plaques. Plaques of this class are white or gray, with thick overlying fibrous caps covering a mass of atheromatous material contained between the fibrous cap and the media. Usually this mass is streaked with yellow and is a solid or semisolid material which contains occasional calcified particles. Rarely, it is semiliquid. On microscopic examination of stained sections, the intimal cap is usually intact, with little or no ulceration, and may be quite thick. Extracellular lipid makes up a major portion of the plaque, and moderately extensive damage to the media is usually present. By ultraviolet light, green fluorescence of the fading and nonfading types is easily found and is scattered in a random manner through all areas which can be stained with Oil Red O. Single sections rarely show an even distribution of this fluorescence; but when serial sections are examined, a random pattern within lipid-bearing areas is the rule. The intensity of the fluorescence varies from bright to dull, and various shades of green may be seen in the same section. Green fluorescence usually occurs as a diffuse, amorphous haze with clumplike areas of increased intensity which fade at various speeds or may not fade at all. Occasionally it appears as small, discrete, irregular bodies which fade. Scattered through the background of amorphous fluorescence are bright, irregular, cuboid bodies, either golden, yellow, or blue. The golden or yellow bodies may be oblong and resemble cholesterol clefts. Decalcification with EDTA imparts a blue tint and appears to diminish areas of green fluores- cence but does not alter the golden, yellow, or blue fluorescent bodies.

Grade IV plaques. These are the most advanced lesions of atherosclerosis. Atheromatous ulcers, necrotic plaques with hemorrhage, and those with large deposits of calcium are included. All show marked medial involvement. Microscopic examination of intact stained sections shows large masses of extracellular lipid, extensive medial damage, and ulceration. With ultraviolet light, these lesions present the most marked fluorescence seen in atherosclerotic plaques. Green fluorescence, bright and dim, fading and nonfading, is present in abundance as an amorphous haze, randomly distributed through the lipid-bearing areas. Cuboid bodies of bright green are seen, often in the neighborhood of clustered, golden, or blue nonfading fluorescent bodies, which are present in greater number than in less advanced lesions. Areas which appear to be in transition from recognizable media to atherosclerotic plaque can be found. Here the medial fibrils are thinner and have lost their definition and brilliance. The normally dark interspaces between fibrils are filled with homogenous green fluorescent material which fades in patches.

Loss of necrotic material in sectioning and the necessity of prolonged decalcification make the examination of Grade IV plaques difficult. EDTA imparts a blue cast to the diffuse background fluorescence, but green, fading fluorescence can be found with ease. Upon occasion, sections were not attempted because calcific plates, semiliquid atheromatous material, and the adventitia were all that remained of the aortic wall. Smears of these lesions revealed green fluorescence in abundance with scattered golden and blue bodies.

\section{B. The identity of green fluorescence in atheromas}

Previously, it has been shown that the yellow pigments extracted from normal and atherosclerotic aortas with ethanol-petrol ether are carotenoids and that these carotenoids will be absorbed onto alumina from hexane and eluted with ethanol-hexane (6). Ethanol-petrol ether extracts of atheromas, yellow by visible light, show a green fluorescence identical with that seen in plaques when dried and examined by ultraviolet microscopy. This examination is made more con- 
venient by placing a few granules of anhydrous sodium sulfate on the slide. The fluorescence of a local concentration of lipids around these granules is more easily found than that of the thin oily film which results when extracts are dried on clean glass. Sodium sulfate granules are dark by ultraviolet light and do not fluoresce until extracts are dried upon them, whereupon they assume the fluorescence of adjacent or adsorbed lipids. After examination, the fluorescent lipid can be recovered by washing with petrol ether, leaving dark granules.

Two experiments are reported in which the fluorescent substances found in atheromas were extracted, chromatographed on alumina, and found to have the mobility of carotenoids and/or vita$\min \mathrm{A}$.

Experiment 1. Approximately one-tenth of a $\mathrm{ml}$. of semiliquid homogenous yellow atheromatous porridge was scooped from the cut surface of an iliac arterial plaque which had a thick overlying fibrous cap and an intact adventitia. A thick smear of this material was opaque to visible light, and one corner of the smear examined by ultraviolet light showed diffuse bright and dim green fluorescence, both fading and nonfading. The smear was extracted with $(10: 100)$ ethanolpetrol ether $\left(30\right.$ to $60^{\circ} \mathrm{C}$.) by gently flowing this solvent over the slide and collecting the effluent. All green fluorescence was immediately removed from the thin areas of the slide, although these areas remained opaque to visible light and the bulk of the material present could be seen to have remained fixed to the slide. Repeated washes left only a few yellow-green particles in the thickest parts of the slide which could not be washed free of fluorescence. The collected washes, reduced to dryness at $20^{\circ} \mathrm{C}$. with water vacuum and taken up in several drops of hexane (Skellysolve B), showed a very faint yellow color. One drop was examined by ultraviolet light on sodium sulfate granules and showed green fluorescence. The remainder was charged onto a 30 by $1 \mathrm{~mm}$. column of alumina prepared in a Pasteur capillary pipette. A sharply defined, strongly adsorbed, pigment band developed which did not move with hexane but immediately passed off the column with ethanol-hexane $(8: 100)$. The ethanol-hexane $(8: 100)$ fraction was collected and when concentrated with water vacuum showed a faint yel-

low color by visible light and green fluorescence when examined on granules of sodium sulfate by ultraviolet light.

Experiment 2. A segment of abdominal aorta showing severe atherosclerosis was stripped of adventitia and assayed by a previously published technique (6). One ml. of an ethanol-petrol ether (10:100) extract of this aorta, containing $2.4 \mu \mathrm{g}$. total carotenoids (39 per cent Fraction I, 44 per cent Fraction II, 17 per cent Fraction III), 16 milligrams total sterols (Lieberman-Burchard reaction) and 24 milligrams total lipid, was charged onto a 10 by $100 \mathrm{~mm}$. column of alumina and the chromatogram developed with acetone-hexane (20:100) until the pigments of Fractions I, II and III were seen to be separated. The chromatogram was then cut into segments, extracted and examined for fluorescence on sodium sulfate granules. From the top of the chromatogram downward the following zones were present:

Zone 1-A $0.25 \mathrm{~cm}$. sharp, yellow band (Fraction III) showed nonfading green fluorescence.

Zone 2-A $1 \mathrm{~cm}$. diffuse, orange band (Fraction II) showed a dimmer, nonfading metallic green fluorescence.

Zone 3-A $2.5 \mathrm{~cm}$. clear zone showed no fluorescence.

Zone 4-A $2 \mathrm{~cm}$. sharp, orange band which was skewed (Fraction I) showed greenish-blue nonfading fluorescence with patches of bright pea-green fluorescence which faded in 20 to 40 seconds.

\footnotetext{
${ }^{3}$ If all carotenoids reported to occur in human tissue were present in an extract subjected to this fractionation, the fractions obtained would contain the following:

Fraction I

Zeta carotene

Alpha carotene

Beta carotene

Fraction II

Prolycopene

Lycopene

An "unknown" carotenoid

Diesters of carotenols found in Fraction III

Fraction $I I I$

Cryptoxanthin

Lutein

Zeaxanthin

Capsanthin

Capsorubin
}

Vitamin A alcohol would migrate with Fraction III ; esterified vitamin A would migrate with Fraction I. 
Zone 5-A $2 \mathrm{~cm}$. colorless zone was nonfluorescent.

Zone 6 -A $2 \mathrm{~cm}$. colorless zone was nonfluorescent.

When the material obtained from all fluorescent zones was recombined and dried upon sodium sulfate, it showed fluorescence of the same shade of green as was present in a sample of unseparated extract.

These experiments provide evidence that essentially all of the green fluorescence in atheromas is extracted with ethanol-petrol ether $(10: 100)$ and, once extracted, all of this fluorescent material behaves on a column of alumina in a manner known to be characteristic of carotenoids. Since vitamin A migrates with carotenoids on the chromatograms described, these experiments do not serve to differentiate the separate contributions of vitamin A and carotenoids to the fluorescence seen in atheromas. Examination of the ultraviolet spectrum of the extract used in Experiment 2, and that from five other aortas, indicates that only relatively little vitamin A can be present in proportion to the concentrations of carotenoids.

\section{DISCUSSION}

It is believed that the chromatographic experiments here reported on the identity of green fluorescence in atheromas, and the previous work of Popper and Greenberg (4) and von Querner (5) on the identity of the green fluorescence in other tissues, make it valid to assume that either carotenoids and/or vitamin A are present in those parts of the atheroma in which green fluorescence is seen. The known functions of carotenoids and vitamin $\mathrm{A}$ in man are vastly different (certain carotenoids can serve as vitamin A precursors, the remainder have no known function), and differences exist in their transport by serum (7) and storage by tissue (8). However, both carotenoids (beta carotene, $\mathrm{C}_{40} \mathrm{H}_{56}$; lutein, $\mathrm{C}_{40} \mathrm{H}_{58} \mathrm{O}_{2}$ ) and vitamin $A\left(\mathrm{C}_{20} \mathrm{H}_{30} \mathrm{O}\right)$ are large lipid molecules which cannot be synthesized by human tissues, and so the distribution of either serves to localize a dietary lipid in the lesions of atherosclerosis. Our findings indicate that dietary lipids of this class are distributed in a random fashion throughout the lipid-bearing areas of atherosclerotic lesions.

Accumulation of carotenoids and/or vitamin A does not appear affected by thick layers of overlying fibrosis nor does it bear any discernible relation to intramural hemorrhage. It is worthy of note that advanced lesions with large lipid masses show more and brighter fluorescence than early, small ones, suggesting that in addition to an increased amount of lipid, late lesions contain an increased concentration of carotenoids and/or vita$\min \mathrm{A}$ in this lipid. This aspect of our study supports the current view that although lipids of early atherosclerotic lesions are quite similar to those found in serum, later lesions show a higher proportion of less polar lipids $(9,10)$, and suggests that selective accumulation of certain serum lipids rather than some other process may be responsible. Certainly, it seems clear that the carotenoid and/ or vitamin A content of the lipid in atheromas are not diluted by the further accumulation of lipid as plaques grow, and this finding by fluorescence microscopy supports the result previously obtained by chemical assay (1).

It is possible that distinction between the presence of carotenoids and vitamin $\mathrm{A}$ in atheromata may assume more importance than is attached to it in this report. Carotenoids, as such, are considered to be relatively inert in human tissue, but vitamin $\mathrm{A}$ and its fluorescent analogues with biologic activity might produce changes in the character of atherosclerotic lesions. Popper and Greenberg have shown that in certain situations differentiation between carotenoids and vitamin A can be made on the basis of intensity of fluorescence and the rate at which it fades (4). However, since the fluorescence of vitamin A may vary with the location in which it is found, its concentration, and the conditions of examination (2), fractionation of the unsaponifiable material from atheromas would seem to be a more reliable means. To date, examination of ultraviolet curves from extracts of six aortas indicates that the vitamin A content must be small in relation to the concentration of carotenoids.

Our findings at present seem applicable only to the continued growth of atheromas and do not shed light on the initiation of plaque formation. It is possible that the factors which initiate atherosclerosis are different from those which cause progression of the lesions. Systematic study of the sequential development of atheromas might suggest some means by which progression may be 
retarded. In view of the fact that most of the pathologic effects of atherosclerosis are associated with the occurrence of advanced lesions rather than early ones, this area of investigation would appear fruitful. One aspect of the change from small, early plaques to large, advanced ones is accumulation of lipid through which carotenoids and/or vitamin $\mathrm{A}$ have been shown to occur at random. Study of the accumulation of carotenoids and vitamin A should help to clarify mechanisms of lipid deposition in advancing atheromas. Further investigation of the fluorescent pattern of atheromas with more sensitive equipment might reveal information unrecognized at present. In addition, the study of carotenemia or hypervitaminosis $\mathrm{A}$ in man and experimental animals should provide a means by which the mechanism of the accumulation of these two exogenous lipids in atheromas may be investigated directly.

\section{CONCLUSIONS}

1. The occurrence of green fluorescence, fading and nonfading, in human atheromas examined microscopically by ultraviolet light is reported.

2. This fluorescence is scattered at random through lipid-bearing areas of plaques and is seen in greater amounts in more advanced lesions.

3. Evidence is presented that this fluorescence is a valid means by which carotenoids and/or vitamin A, two lipids of dietary origin, can be localized in the lesions of atherosclerosis.

4. These findings do not appear to apply to present theories regarding the initiation of atheromas. They suggest that increase in size and severity of atherosclerotic lesions is accompanied by a selective accumulation of certain nonpolar lipids, some of which are derived from diet.

\section{ACKNOWLEDGMENT}

Grateful acknowledgment is made to Mrs. Elizabeth C. Wise for aid in preparation of the manuscript.

\section{REFERENCES}

1. Blankenhorn, D. H., Freiman, D. G., and Knowles, H. C., Jr. Carotenoids in man. I. The distribution of epiphasic carotenoids in atherosclerotic lesions. J. clin. Invest. 1956, 35, 1243.

2. Popper, H. Histologic distribution of vitamin A in human organs under normal and under pathologic conditions. Arch. Path. 1941, 31, 766.

3. Hamperl, H. Die Fluorescenzmikroskopie menschlicher gewebe. Virchows Arch. 1934, 292, 1.

4. Popper, H., and Greenberg, R. Visualization of Vitamin $\mathrm{A}$ in rat organs by fluorescence microscopy. Arch. Path. 1941, 32, 11.

5. von Querner, F. R. Der mikroskopische Nachweis von Vitamin A im animalen gewebe. Klin. Wschr. 1935, p. 1213, II Haljahr.

6. Blankenhorn, D. H. Carotenoids in man. II. Fractions obtained from atherosclerotic and normal aortas, serum, and depot fat by separation on alumina. J. biol. Chem. 1957, 227, 963.

7. Krinsky, N. I., Cornwell, D. G., and Oncley, J. L. Vitamin A, carotenoids and plasma lipoproteins. Fed. Proc. 1956, 15, 113.

8. Clausen, S. W. The absorption of Vitamin A and its storage in the tissues. Harvey Lect. Series 38. Lancaster, The Science Press Printing Co., 1943. p. 199.

9. Hirsch, E. F. The chemistry of atherosclerosis in man and its pathogenesis as indicated by animal experiments. Trans. Amer. ther. Soc. 1948, 48, 113.

10. Page, I. H. Atherosclerosis. An introduction. Circulation 1954, 10, 1. 